\title{
Associação entre o uso excessivo de álcool e fibrilação atrial em tempos de pandemia da Covid-19: uma investigação necessária
}

\author{
Association between excessive alcohol use \\ and atrial fibrillation in times of Covid-19 \\ pandemic: a necessary investigation
}

\section{Guilherme Brasil Grezzana' ${ }^{1}$ Airton Tetelbom Stein ${ }^{2}$ (1)}

\author{
1Autor para correspondência. Clínica Del Cuore (Antônio Prado). Rio Grande do Sul, Brasil. gbgrezzana@yahoo.com.br \\ Universidade Federal de Ciências da Saúde de Porto Alegre (Porto Alegre). Rio Grande do Sul, Brasil. airton.stein@gmail.com
}

A atual pandemia pelo covid-19 tem sido uma experiência social global inusitada com diversas implicações quanto aos cuidados em saúde. ${ }^{1} \mathrm{~A}$ descrição da COVID-19 mostra uma interação entre doenças dentro das características das populações específicas. Estas condições estão se agrupando dentro de grupos sociais de acordo com padrões de iniquidade. A interação entre essas doenças em um contexto de disparidade social e econômica exacerba os efeitos adversos de cada doença. A COVID-19 não é uma pandemia. É uma sindemia.?

A pandemia COVID-19 indica que a elevada frequência de problemas de saúde mental seja caracterizada na lista de prioridades de saúde global. $\frac{3}{} \mathrm{O}$ aumento nos diagnósticos de depressão e ansiedade sugerem um impacto significativo da pandemia por Covid-19 em relação à prevalência de estresse psicológico. ${ }^{4}$ É relevante destacar que medidas coercitivas, como o isolamento social ou quarentena, na tentativa de impedir o avanço do covid-19, podem suscitar o surgimento ou exacerbação de sentimentos negativos de depressão, manifestações de raiva, culpa e medo. ${ }^{5}$ Dessa forma, algumas pessoas em isolamento social e estresse psicológica relacionada a pandemia, podem desencadear comportamentos não saudáveis como uso abusivo de álcool. ${ }^{4}$ Somam-se a esses fatores, as incertezas quanto a manutenção ou obtenção de empregos e as ameaças econômicas como gatiIho para a ingestão de bebidas alcóolicas. $\frac{6}{-}$

O uso inadequado da ingestão de álcool contribui para cerca de três milhões de mortes diretas em todo o mundo além da associação com outros fatores de risco para desfechos clínicos importantes. ${ }^{6} \mathrm{~A}$ utilização abusiva do álcool leva à neuroadaptações que aumentam a necessidade do uso do álcool em um sistema de retroalimentação. ${ }^{6}$ Devemos levar em consideração as diferenças individuais nos efeitos recompensadores e desinibidores do álcool, onde as respostas agudas à utilização de bebidas alcóolicas podem gerar efeitos dinâmicos quanto a tolerância e necessidades de aumento do consumo. ${ }^{-}$Assim, todos esses ingredientes abrem espaço para o uso abusivo do álcool e substâncias que acionam mecanismos de recompensa e alívio transitório de sentimentos negativos $\stackrel{8}{ }$, que também podem ser desencadeantes de desordens psiquiátricas., $\frac{89}{9}$ 
A fibrilação atrial é uma arritmia que aumenta a sua prevalência de acordo com o envelhecimento populacional ${ }^{1,10}$ e denota maior risco para desfechos cardiovasculares como acidente vascular cerebral e insuficiência cardíaca. ${ }^{11} \mathrm{~A}$ ingestão de bebidas alcóolicas apresenta efeitos arritmogênicos tanto durante o consumo agudo ${ }^{12,13}$ quanto ao consumo crônico, esse através de mudanças na estrutura cardíaca. ${ }^{6.11} \mathrm{~A}$ ingestão habitual de bebidas alcóolicas está relacionada ao remodelamento atrial bem como associada a prevalência de outros fatores de risco para fibrilação atrial. 11 Esses dados foram observados em estudos epidemiológicos ${ }^{10,11}$ independentemente do tipo de bebida alcóolica ingerida, onde a fibrilação atrial já pode ser observada a partir de pequenas doses quotidianas de álcool (acima de $2 \mathrm{~g} / \mathrm{dia}) .{ }^{11}$

Estudo de coorte com base comunitária1ㅜㄹ demonstrou riscos crescentes para fibrilação atrial relacionados ao consumo de bebidas alcóolicas. Esses achados, são respaldados pela relação entre abstinência e redução de fibrilação atrial. ${ }^{14}$ Dessa forma, devem ser priorizadas informações corretas ao público geral quanto aos malefícios do álcool, especialmente relacionado ao atual momento de enfrentamento ao COVID-19, no que consta na falsa premissa protetora da ingesta controlada e protetora de bebidas alcoólicas ${ }^{15}$ e considerando um amento de até $29 \%$ no consumo de álcool desde o início da epidemia. ${ }^{16}$

Algumas medidas na tentativa de conter a epidemia global do Covid-19, como o isolamento social, geraram demandas de cuidados em saúde e, dentre essas, o uso abusivo de bebidas alcóolicas. $\stackrel{-}{-}$ Há necessidade premente de suporte das diversas sociedades, familiares, sistemas de saúde em estado de colaboração mútuo com os seus respectivos governos e divisões de responsabilidades.. O monitoramento dos níveis de consumo durante e após a pandemia da COVID-19 será necessário para entender melhor os efeitos da Covid-19 em diferentes grupos, bem como para distingui-los daqueles decorrentes das políticas existentes de controle do álcool. Os médicos e profissionais de saúde devem abordar sobre a mudança de hábito de consumo de álcool durante a pandemia e orientarem atividades de promoção de saúde com o intuito de esclarecer os danos potenciais do uso do álcool para doenças cardiovasculares, especialmente a fibrilação atrial.

\section{Contribuições dos autores}

Todos os autores contribuíram ativamente para a elaboração do relato e aprovação da versão final.

\section{Conflitos de interesses}

Nenhum conflito financeiro, legal ou político envolvendo terceiros (governo, empresas e fundações privadas, etc.) foi declarado para nenhum aspecto do trabalho submetido (incluindo, mas não se limitando a subvenções e financiamentos, participação em conselho consultivo, desenho de estudo, preparação de manuscrito, análise estatística, etc.).

\section{Referências}

1. Rehm J, Kilian C, Ferreira-Borges C, Jernigan D, Monteiro $\mathrm{M}$, Parry CDH, et al. Ahcohol use in times of the COVID 19: Implications for monitoring and policy. Drug Alcohol Rev. 2020;39(4):301-4. https://doi.org/10.1111/dar.13074

2. Horton R. Offline: COVID-19 is not a pandemic. Lancet. 2020;396(10255):874. https://doi.org/10.1016/s01406736(20)32000-6

3. The Lancet Global Health. Mental health matters. Lancet Glob Health. 2020;8(11):e1352. https://doi.org/10.1016/s2214$109 \times(20) 30432-0$

4. Stanton R, To QG, Khalesi S, Williams SL, Alley SJ, Thwaite $\mathrm{TL}$, et al. Depression, Anxiety and Stress during COVID-19: Associations with Changes in Physical Activity, Sleep, Tobacco and Alcohol Use in Australian Adults. Int J Environ Res Public Health. 2020;17(11):4065. https://doi.org/10.3390/ijerph17114065

5. Brooks SK, Webster RK, Smith LE, Woodland L, Wessely S, Greenberg N, et al. The psychological impact of quarantine and how to reduce it: rapid review of the evidence. Lancet. 2020;395(10227):912-20. https://doi.org/10.1016/s01406736(20)30460-8

6. Clay JM, Parker MO. Alcohol use and misuse during the COVID-19 pandemic: a potential public health crisis? Lancet Public Health. 2020;5(5):e259. https://doi.org/10.1016/s24682667(20)30088-8

7. Allen HC, Weafer J, Wesley MJ, Fillmore MT. Acute rewarding and disinhibiting effects of alcohol as indicators of drinking habits. Psychopharmacology (Berl). 2021;238(1):181-91. https://doi. org/10.1007/s00213-020-05667-w

8. Dubey J.J, Ghosh R, Chatterjee S, Biswas P, Chatterjee S, Dubay S. COVID-19 and addiction. Diabetes Metab. Syndr.: Clin. Res. Rev. 2020;14(5):817-23. https://doi.org/10.1016/j.dsx.2020.06.008 
9. Ornell F, Moura HF, Scherer JN, Pechansky F, Kessler FHP, von Diemen L. The COVID-19 pandemic and its impact on substance use: Implications for prevention and treatment. Psychiatry Res. 2020;289:113096. https://dx.doi.org/10.1016\%2Fj. psychres.2020.113096

10. Kodama S, Saito K, Tanaka S, Horikawa C, Saito A, Heianza $Y$, et al. Alcohol consumption and risk of atrial fibrillation: a meta-analysis. J Am Coll Cardiol. 2011;57(4):427-36. https://doi. org/10.1016/j.jacc.2010.08.641

11. Csengeri D, Sprünker NA, Di Castelnuovo A, Niiranen T, Vishram-Nielsen JK, Costanzo S, et al. Alcohol consumption, cardiac biomarkers, and risk of atrial fibrillation and adverse outcomes. Eur Heart J. 2021;42(12):1170-7. https://doi. org/10.1093/eurheartj/ehaa953

12. Brunner S, Herbel R, Drobesch C, Peters A, Massberg S, Kääb $S$, et al. Alcohol consumption, sinus tachycardia, and cardiac arrhythmias at the Munich Octoberfest: results from the Munich Beer Related Electrocardiogram Workup Study (MunichBREW). Eur Heart J. 2017;38(27):2100-6. https://doi.org/10.1093/eurheartj/ ehx156
13. Voskoboinik A, Prabhu S, Ling LH, Kalman JM, Kistler PM. Alcohol and Atrial Fibrillation: A Sobering Review. J Am Coll Cardiol. 2016;68(23):2567-76. https://doi.org/10.1016/j. jacc.2016.08.074

14. Dixit S, Alonso A, Vittinghoff E, Soliman EZ, Chen LY, Marcus GM. Past alcohol consumption and incident atrial fibrillation: The Atherosclerosis Risk in Communities (ARIC) Study. PLoS One. 2017;12(10):e0185228. https://doi.org/10.1371/journal. pone. 0185228

15. Sharma A, Kroumpouzos G, Lotti T, Goldust M. COVID-19 and alcohol use. Drug Alcohol Rev. 2021;40(4):683-4. https://doi. org/10.1111/dar.13163

16. Capasso A, Jones AM, Ali SH, Foreman J, Tozan Y, DiClemente RJ. Increased alcohol use during the COVID-19 pandemic: The effect of mental health and age in a cross-sectional sample of social media users in the U.S. Prev Med. 2021;145:106422. https:// doi.org/10.1016/j.ypmed.2021.106422 